\title{
Diffusion inélastique sur des composés magnétiques
}

\author{
B. Dorner
}

Institut Laue-Langevin, BP. 156x, 38042 Grenoble cedex, France

\section{INTRODUCTION}

Ce cours est le second d'une série de trois. L'approche théorique de la diffusion magnétique et les techniques expérimentales ont été développées par Björn Fåk.

Món exposé commence par une brève introduction sur la résolution expérimentale en insistant sur les spectromètres trois axes (TAS). Ensuite, deux exemples de détermination des courbes de dispersion des magnons sont présentés : La chaîne ferromagnétique unidimensionnelle (1-D) $\mathrm{CsNiF}_{3}$ et le grenat antiferromagnétique 3- $\mathrm{D} \mathrm{Ca}_{3} \mathrm{Fe}_{2}\left(\mathrm{GeO}_{4}\right)_{3}$.

Après une approche générale des systèmes ferro- et antiferromagnetiques, de nouveaux développements pour certains systèmes antiferromagnétiques sont expliqués. Les Spinons, excitations dans des systèmes $S=1 / 2$ ont été prédits et observés. Les résultats expérimentaux sur $\mathrm{CuGeO}_{3}$ obtenus en temps de vol (TOF) avec le spectromètre MARI installé sur la source pulsée ISIS montre que le TOF est particulièrement utile pour les systèmes 1-D.

A la fin, les excitations dans les composés de type $\mathrm{CsFeCl}_{3}$ présentant un état singulet fondamental sont discutées. Ces excitations sont appelées excitons car elles ont un moment net nul dans l'état fondamental.

\section{LA RÉSOLUTION EN DIFFUSION DE NEUTRONS INÉLASTIQUE}

La relation entre la section efficace doublement différentielle et la fonction de structure $S(Q, \omega)$ est donnée par :

$$
\frac{d^{2} \sigma}{d \Omega d \omega}=\frac{k_{F}}{k_{l}} S(\mathbf{Q}, \omega)
$$

d $\Omega$ est l'angle solide dans lequel les neutrons sont diffusés. $\omega$ et d $\omega$ sont le transfert d'énergie et sa résolution. $\mathbf{Q}=\mathbf{k}_{\mathrm{l}}-\mathbf{k}_{\mathrm{F}}$ est le vecteur de diffusion, où $\mathbf{k}_{\mathrm{l}}$, $\mathbf{k}_{\mathrm{F}}$ sont les vecteurs d'onde incident et diffusé du neutron. 
L'intensité diffusée, $\Delta \mathrm{I}[1]$ est

$$
\Delta I=\phi_{0} N \frac{d^{2} \sigma}{d \Omega d \omega} p_{F}\left(k_{F}\right) \Delta \Omega \Delta \omega
$$

$\phi_{0}$ est l'intensité đu faisceau monochromatique, $\mathrm{p}_{\mathrm{F}}\left(\mathbf{k}_{\mathrm{F}}\right)$ la transmission de l'analyseur et $\mathrm{N}$ le nombre de particules diffusantes (volume de l'échantillon).

$$
\phi_{0}=A\left(\boldsymbol{k}_{i}\right) \cdot \boldsymbol{k}_{I} \cdot p_{i}\left(\boldsymbol{k}_{l}\right) \Delta \boldsymbol{k}_{i, x} \cdot \Delta \boldsymbol{k}_{i, y} \cdot \Delta \boldsymbol{k}_{t, z}
$$

où $A\left(\mathbf{k}_{\mathrm{I}}\right)$ correspond au spectre de la source de neutrons et $\mathrm{p}_{\mathrm{f}}\left(\mathbf{k}_{\mathrm{l}}\right)$ est la transmission du monochromateur.

On a

$$
\Delta \Omega=\frac{\Delta k_{F, x} \cdot \Delta k_{F, y}}{k_{F}^{2}} ; \Delta \omega=\frac{\hbar}{m} k_{F} \Delta k_{F, z}
$$

et donc

$$
\Delta I=L \cdot S(\boldsymbol{Q}, \omega) \cdot p_{l}\left(\boldsymbol{k}_{l}\right) \Delta V_{l} \cdot p_{F}\left(\boldsymbol{k}_{F}\right) \Delta V_{F}
$$

Les volumes élémentaires dans l'espace réciproque sont :

$$
\Delta V_{l}=\Delta k_{l, x} \cdot \Delta k_{l, y} \cdot \Delta k_{i, z} ; \Delta V_{F}=\Delta k_{F, x} \cdot \Delta k_{F, y} \cdot \Delta k_{F, z}
$$

$L$ contient le spectre du réacteur et le volume de l'échantillon.

L'Eq. (5) montre clairement que $S(Q, \omega)$ décrit mieux la diffusion que la section efficace.

Le principal avantage réside dans le fait que les conditions expérimentales, c'est-à-dire l'instrument et sa résolution apparaissent de façon symétrique pour le monochromateur $\left(\mathrm{p}_{\mathrm{l}}\left(\mathrm{k}_{\mathrm{l}}\right) \Delta \mathrm{V}_{\mathrm{l}}\right)$ et l'analyseur $\left(\mathrm{p}_{\mathrm{F}}\left(\mathbf{k}_{\mathrm{F}}\right) \Delta \mathrm{V}_{\mathrm{F}}\right)$. Le produit des deux volumes $\Delta \mathrm{V}_{\mathrm{I}} \cdot \Delta \mathrm{V}_{\mathrm{F}}$ détermine l'intensité transferée possible. $\mathrm{L}$ 'intensité réelle mesurée dépend bien entendu de $S(\mathbf{Q}, \omega)$.

La convolution des deux volumes élémentaires donnent la résolution de l'appareil. Dans un montage optimum, $\Delta V_{1}$ et $\Delta V_{F}$ ont des tailles comparables.

Dans le cas où la monochromaticité et l'analyse sont faites par des monocristaux, ces volumes élémentaires sont donnés par [1]:

$$
\Delta V_{l} \sim k_{l}^{3} \cot \theta_{M} ; \quad \Delta V_{F} \sim k_{F}^{3} \cot \theta_{A}
$$

où $\theta_{\mathrm{M}}$ et $\theta_{\mathrm{A}}$ sont les angles de Bragg du monochromateur et de l'analyseur.

\section{RÉSOLUTION EN TROIS AXES, TAS}

Dans la technique trois axes, les énergies incidentes et diffusées sont déterminées par des réflexions de Bragg sur de gros monocristaux, souvent composites. La relation de Bragg s'écrit

$$
2 k \sin \theta=n \cdot \tau
$$


$k=2 \pi / \lambda$ est le vecteur d'onde des neutrons, $\theta$ l'angle de reflexion, $n=1,2$ l'ordre de la réflexion et $\tau$ un vecteur de l'espace réciproque, décrivant les réflexions sur des séries de plans [2].

La Figure 1 donne une vision graphique de l'équation (8). En oubliant le rôle des collimateurs avant et après le cristal, la distribution des neutrons diffusés est, de façon évidente, globalement parallèle aux plans réfléchissants. Dans ce sens et moyennant une approximation Gaussiènne, $\Delta \mathrm{V}_{1}$ et $\Delta V_{\mathrm{F}}$ peuvent être définis comme des ellipsoïdes tri-dimensionnelles autour de $\mathbf{k}_{\mathrm{I}}$ et $\mathbf{k}_{\mathrm{F}}$ avec une inclinaison par rapport à ces derniers proche de l'angle de Bragg.

Afin de déterminer la résolution ou la fonction de transmission d'un trois axes, on doit convoluer les deux ellipsoïdes $\Delta \mathrm{V}_{\mathrm{I}}$ et $\Delta \mathrm{V}_{\mathrm{F}}$. On obtient alors une ellipsoïde à 4 dimensions dans l'espace $\mathrm{Q}-\omega$.
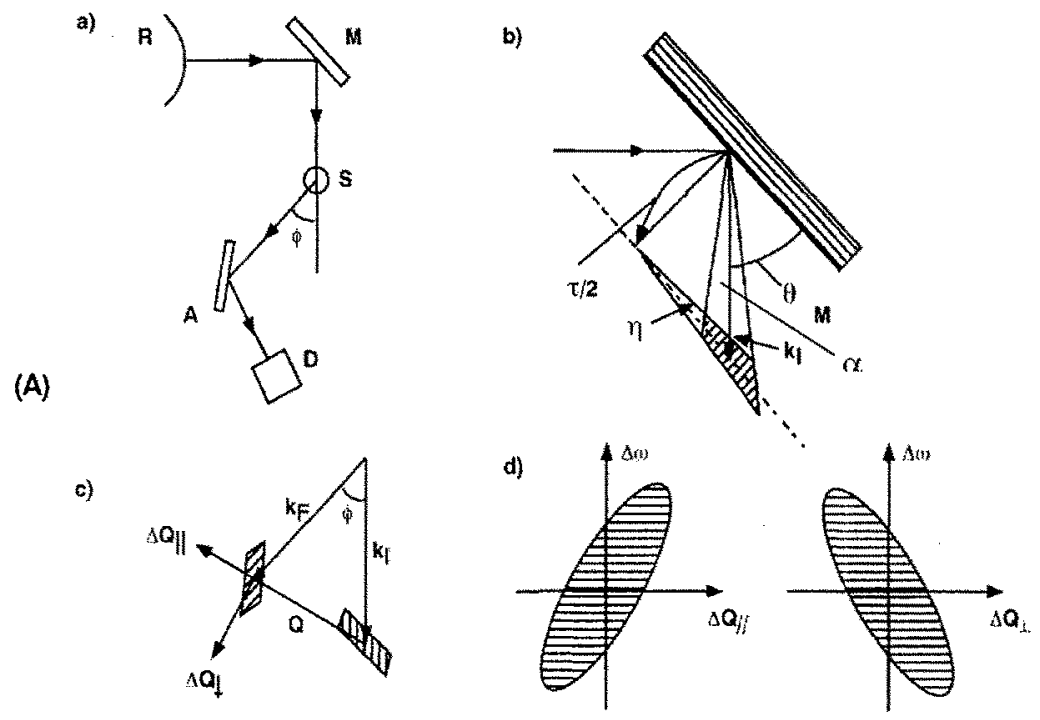

Figure 1: Résolution et transmission d'un triple axe, partie I.

$R, M, S, A$ and $D$ signifient: réacteur, monochromateur, échantillon, analyseur et detecteur. La réflexion de Bragg d'un monocristal monochromateur est montrée en b), où $\theta_{M}$ est l'angle de Bragg et $\tau$ le vecteur correspondant dans l'espace réciproque. $\eta$ est la mosäicité, $\alpha$ la collimation du faisceau réfléchi et la zone hachurée montre la distribution des neutrons diffusés correspondant $\grave{a} k_{\text {. }}$.

Le TAS a esssentiellement quatre géométries différentes. Après avoir fixé une direction de réflexion sur le monochromateur, la diffusion sur l'échantillon peut se faire vers la gauche ou la droite et, de même, la réflexion sur l'analyseur peut se faire à gauche ou à droite. Ces quatre géométries sont présentées figure 2 [3]. La projection de l'ellipsoïde sur les plans $Q_{\|}-\omega$ et $\mathrm{Q}_{\perp}-\omega$ montre les variations possibles. La distribution suivant $Q_{z}$, perpendiculaire au plan de diffusion est toujours perpendiculaire aux trois composantes $Q_{\|}, Q_{\perp}, \omega$.

Il y a un effet de focalisation, si la pente de l'ellipsoïde de résolution a le même sens que la pente de la courbe de dispersion étudiée.

Empiriquement, la focalisation pour les modes transverses acoustiques avec perte d'énergie pour les neutrons apparaît toujours si le vecteur d'onde du phonon $q$ a une direction opposée à $\mathbf{k}_{\mathrm{F}}$.

Pour des études précises, des programmes sont disponibles sur les spectromètres trois axes. 
(B)<smiles>[R]C(C)C(C)C([R])C</smiles>

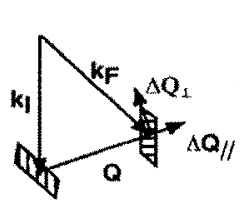
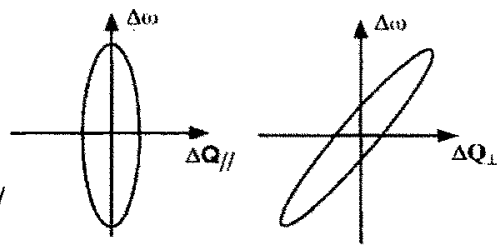

(C)<smiles>[M]C(C)C(C)C</smiles>
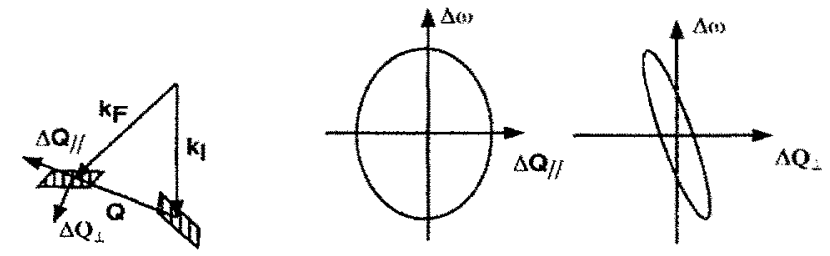

(D)

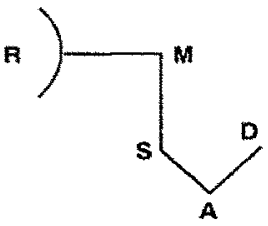

(a)

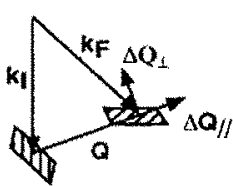

(c)

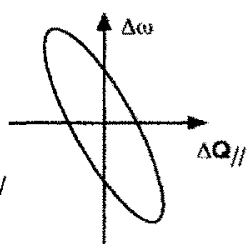

(d)

Figure 2: Résolution et transmission d'un trois axes, partie II.

Les symboles ont la même signification que pour la figure 1. Les ellipses representent les projections des ellipsoïdes de résolution à 4-dimensions sur les plans $Q_{i r} \sim \omega_{\text {et }} Q_{r} \sim \omega$.

\section{$4 \mathrm{CsNiF}_{3}$}

Ma pemière expérience sur des excitations magnétiques a conduit à la première publication d'un travail expérimental à l'ILL [4]. Nous avons étudié le ferromagnétique uni-dimensionnel $\mathrm{CsNiF}_{3}$. La figure 3 montre la structure de ce composé. Le caractère remarquable de cette structure est la présence de chaînes fortement couplées de $\mathrm{Ni}^{2+}(\mathrm{S}=1)$ séparées par des octaèdres de $\mathrm{NiF}_{6}$ partageant une face. La distance $\mathrm{Ni}-\mathrm{Ni}$ le long des chaînes est de $2.6 \AA$ et la distance entre chaînes est $6.2 \AA$. L'interaction magnétique entre chaines est de plusieurs ordres de grandeur plus faible que le long des chaînes. $\mathrm{CsNiF}_{3}$ s'ordonne à $T_{N}=2.8 \mathrm{~K}$, les chaînes ferromagnétiques se couplant antiferromagnétiquement entre elles. 

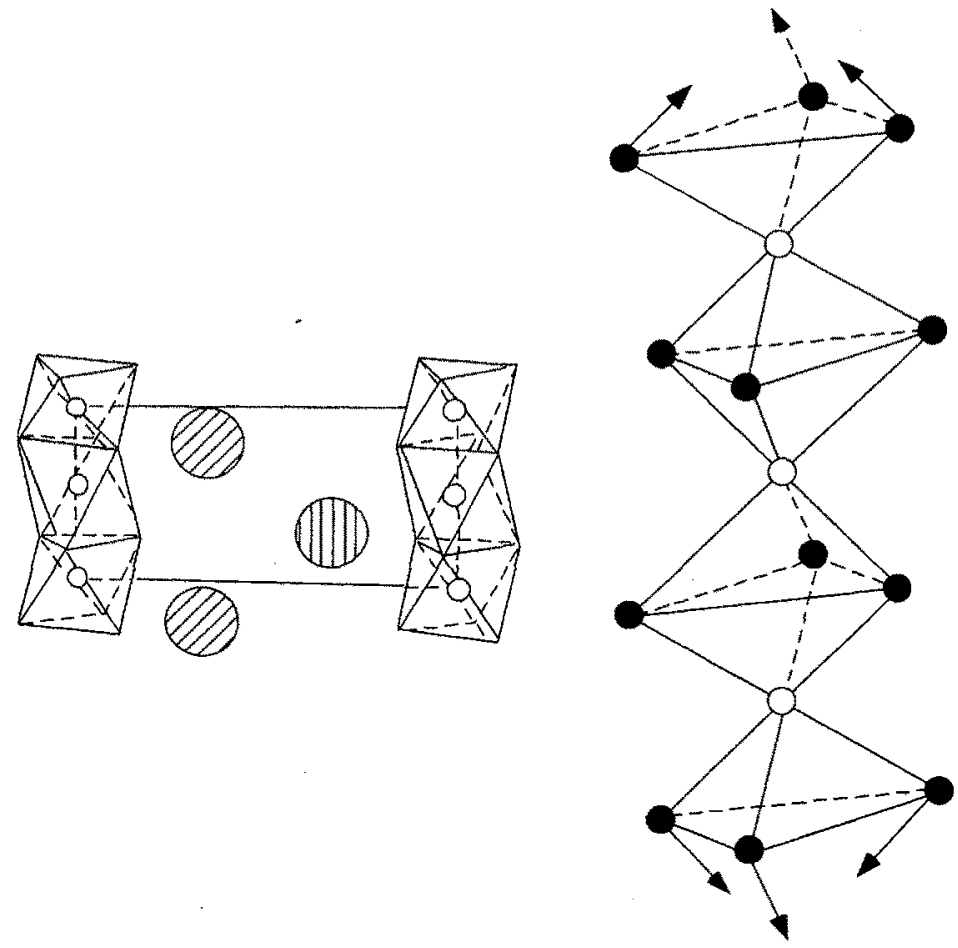

Figure 3: La structure de $\mathrm{CsNiF}_{3}$, comme celle de $\mathrm{CsFeCl}_{3}$ et $\mathrm{CsFeBr}_{3}$, consiste en chaînes d'octaèdres $\mathrm{NiF}_{6}$ partageant une face dans la direction $c$, séparée par les ions $C$.

Lors du premier essai de mesure de la courbe de dispersion des excitations magnétiques à $4.2 \mathrm{~K}$ ( dans le régime 1D) sur le trois axes IN2, nous n'avons pas pu mesurer de signal inélastique.

Le monocristal était relativement petit mais l'effet principal venait de la résolution. L'ellipsoïde de résolution était tellement parallèle à la pente de la courbe de dispersion que le signal, lors de mesures à $Q$ constant, avait une largeur en énergie de $0.16 \mathrm{meV}$, alors que la résolution en énergie totale était de l'ordre de $1 \mathrm{meV}$. Finalement, avec des pas en énergie plus petits, nous avons trouvé le signal et mesuré la courbe de dispersion. La figure 4 montre aussi qu'il n'y a pas de variation de fréquence observable le long de $q_{a}$, qui est perpendiculaire à la direction de la chaîne. Cela était attendu pour un système $1 \mathrm{D}$. 

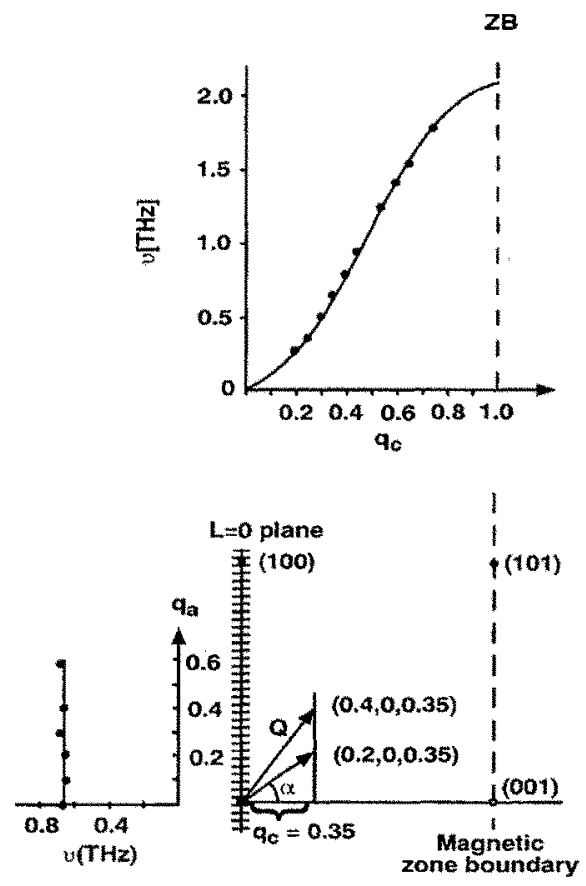

Figure 4: La courbe de dispersion des magnons $\mathrm{CsNiF}_{3}$ le long de la direction cà $4.2 \mathrm{~K}$.

Perpendiculairement à la chaîne, (voir la partie basse), aucune variation de fréquence n'a été observée pour ce système 1-D.

Nous avons aussi analysé les intensités intégrées. Comme le montre la figure 5 , elles sont indépendantes de $\mathrm{q}_{\mathrm{c}}$ et donc de la fréquence de l'excitation. Ceci est attendu pour la diffusion de neutrons sur des magnons dans de simples ferromagnétiques [5].

Le Hamiltonien pour $\mathrm{CsNiF}_{3}$ s'écrit [6]

$$
H=-2 J \sum_{i} S_{i} S_{i+1}+D \sum_{i}\left(S_{i}^{z}\right)^{2}
$$

où $J$ est le paramètre d'échange entre proche voisins le long des chaînes et $D$ rend compte de l'anisotropie locale qui confine les spins dans le plan $x-y$.

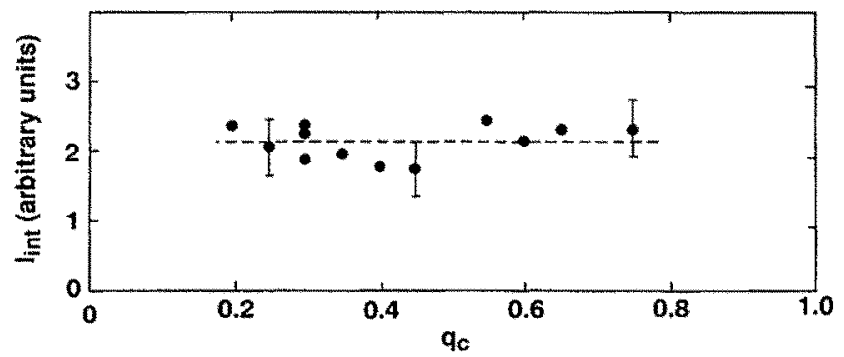

Figure 5: Intensités intégrées et corrigées par la résolution des magnons mesurés dans $\mathrm{CsNiF}_{3}$. 
La courbe de dispersion qui en résulte est donnée par :

$$
\hbar \omega=4 S\left[\left(2 J-2 J \cos \left(\pi q_{c}\right)\left(2 J-2 J \cos \left(\pi q_{c}\right)+D\right)\right] / 2\right.
$$

Cette expression a été ajustée aux résultats expérimentaux avec $J=5.50 \pm 0.02 \mathrm{meV}$ et $\mathrm{D}=0.41 \pm 0.05 \mathrm{meV}$ (figure 4).

Un système $1 \mathrm{D}$ a, à température finie, une longueur de corrélation finie qui ne tend vers l'infini qu'à température nulle. Cette longueur de corrélation finie se traduit par une largeur en énergie finie pour les excitations (Fig. 6a). L'élargissement en fonction de la température est bien expliqué théoriquement[6].
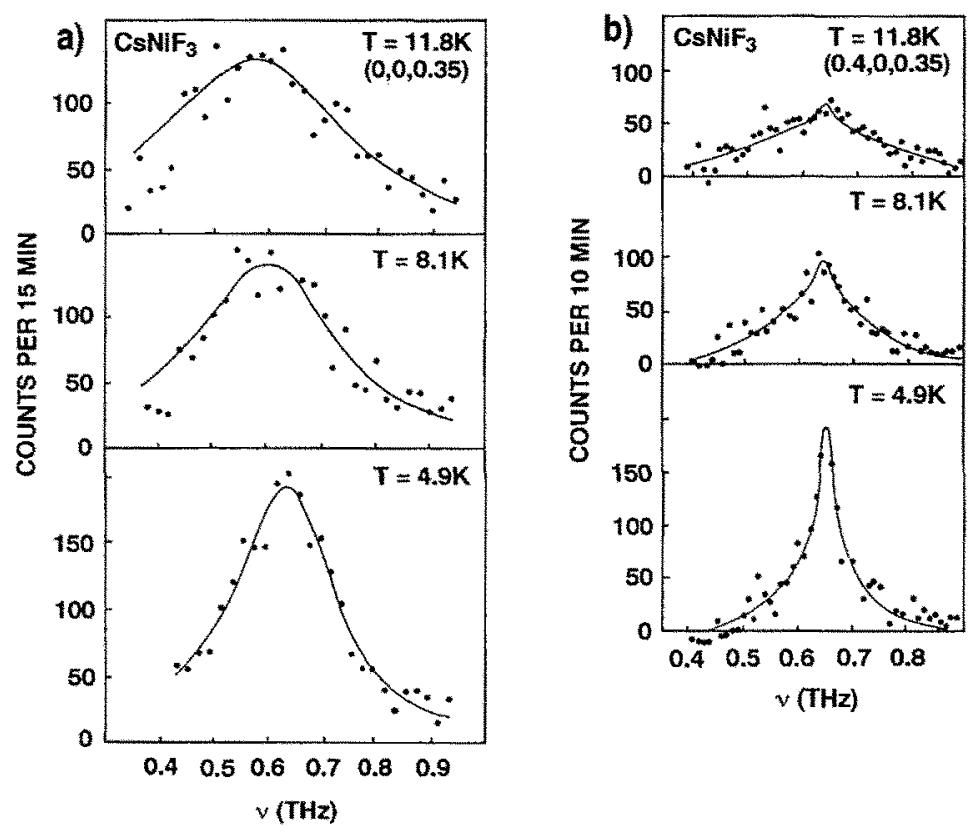

Figure 6: a) Elargissement des signaux provenant des fluctuations $x-y$ dans $C s N i F_{3 .}$ Les lignes continues représentent des ajustements Lorentziens. b): Signaux identiques, mais à une autre valeur de $Q$ choisie de telle sorte que les fluctuations $x-y$ (larges, ajustées par des Lorentziennes) et les fluctuations le long de z(étroites, ajustées par des Gaussiennes) apparaissent simultanément.

Les signaux de la figure $6 \mathrm{a}$ ont été mesurés avec $\mathbf{Q}$ parallèle à l'axe $\mathrm{c}$. Donc uniquement les fluctuations magnétiques le long de $\mathrm{x}$ et $\mathrm{y}$ sont observables. Pour observer la composante $z$ des fluctuations, il faut que $\mathbf{Q}$ ait une composante perpendiculaire à $\mathbf{z}$. De telles mesures sont données figure $6 \mathrm{~b}$. On peut y voir qu'un signal étroit (limité par la résolution) apparaît, lorsque $\mathrm{q}_{\mathrm{a}}$ augmente, sur le sommet du large signal des composantes $\mathrm{x}$ et $\mathrm{y}$. Cela montre bien que la longueur de 
corrélation de la composante $z$ est plus grande que celle des deux autres composantes. L'explication de cet effet [6], confirmé plus tard à l'aide de neutrons polarisés [7], est que l'anisotropie D confine les spins dans le plan $x-y$ de telle sorte que la partie statique de l'orientation des spins a une valeur nulle le long de la direction $z$. Dit autrement, la corrélation des fluctuations suivant $z$ n'est pas réduite par la partie statique, contrairement aux composantes $x$ et $y$.

$\mathrm{CsNiF}_{3}$ est devenu un système 1-D modèle et la recherche sur ce système a continué pendant plus de 20 ans après 1973, en regardant notamment d'autres problèmes plus exotiques comme les "solitons".

\section{LE GRENAT $\mathrm{Ca}_{3} \mathrm{Fe}_{2}\left(\mathrm{GeO}_{4}\right)_{3}$}

La structure chimique de $\mathrm{Ca}_{3} \mathrm{Fe}_{2}\left(\mathrm{GeO}_{4}\right)_{3}$ est cubique centrée avec un paramètre de maille de $\mathrm{a}=12.32 \AA$. La maille primitive magnétique est donnée par le cube de côté $\mathrm{a}=12.32 \AA$. Elle est deux fois plus grande que la maille primitive chimique non cubique et contient $16 \mathrm{Fe}^{3+}(\mathrm{S}=5 / 2)$ ions. L'ordre antiferromagnétique est donné Figure 7. Les deux sous-réseaux sont donnés par des cercles et des carrés.

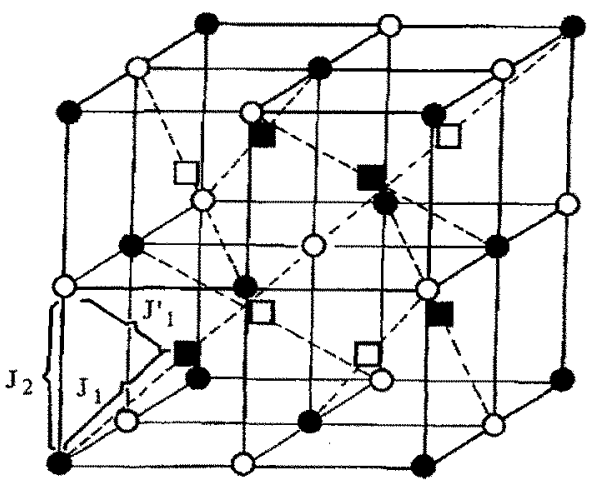

Figure 7: Dans $\mathrm{Ca}_{3} \mathrm{Fe}_{2}\left(\mathrm{GeO}_{4}\right)_{3}$ les ions $\mathrm{Fe}^{3+}$ sont arrangés en deux sous-systèmes décalés de (1/4 1/4 1/4): 0 sont les spins up et down du premier sous-système et $\square$ sont les spins up et down du second. Les J representent les interactions d'échange et les lignes pointillées indiquent les axes de symétrie d'ordre 3 de la structure grenat

En tant qu'illustration simple des ondes de spin, on peut oublier les détails de la structure grenat. Chaque sous-système peut être interprété comme deux sous-réseaux FCC avec quatre spins up et quatre spins down déplacés de $(1 / 200)$. Le second sous-système est en $(1 / 41 / 41 / 4)$ par rapport au premier. Cette vision en FCC aide beaucoup pour l'expérience [8]. La figure 8 montre la zone de Brillouin du cubique simple et du FCC qui est 4 fois plus grande(deux fois dans le plan). 


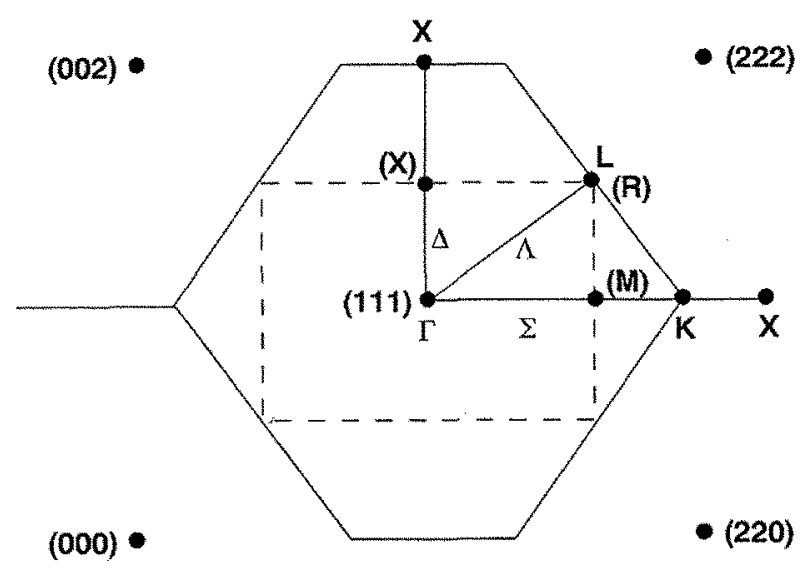

Figure 8: La zone de Brillouin de $\mathrm{Ca}_{3} \mathrm{Fe}_{2}\left(\mathrm{GeO}_{4}\right)$ dans l'espace réciproque. (111) est une position de Bragg antiferromagnetique. (220) et (222) sont des positions de Bragg nucléaires. (002) est éteinte. Les lignes continues correspondent à la maille élémentaire d'un FCC, tandis que la zone hachurée représente la vraie zone de Brillouin.

Les courbes de dispersion calculées et mesurées, des ondes de spin sont montrées figure 9. Elles sont doublement dégénérées.
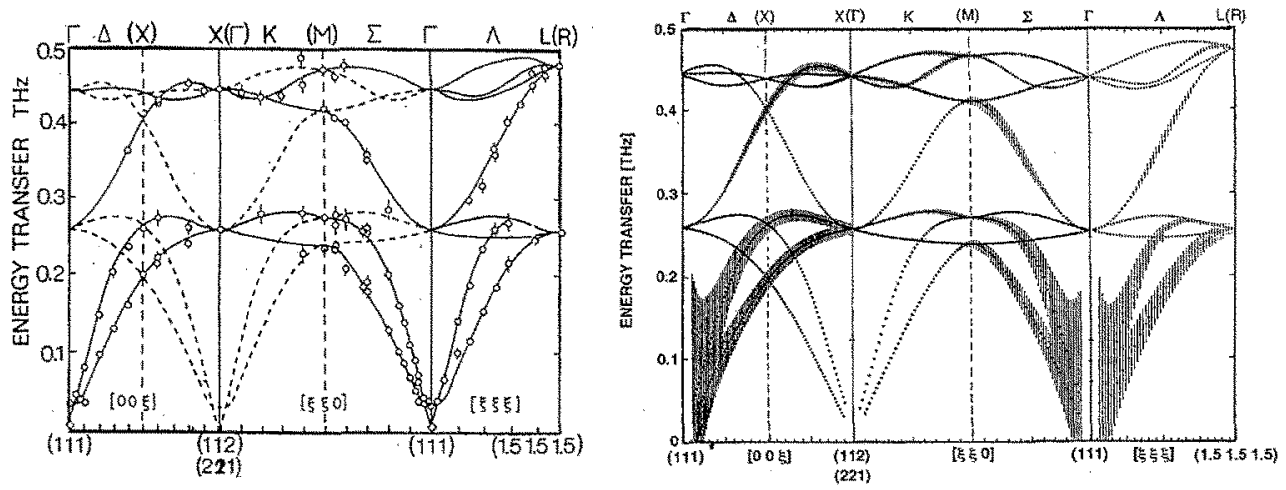

Figure 9: Courbe de dispersion des Magnons pour $\mathrm{Ca}_{3} \mathrm{Fe}_{2}\left(\mathrm{GeO}_{4}\right)_{3}$. La partie gauche donne les résultats expérimentaux (ronds ouverts) et la partie droite, les calculs. Les lignes continues correspondent à la maille élémentaire d'un $F C C$, tandis que la zone hachurée représente la vraie zone de Brillouin. Les intensités prédites, à droite, montrent l'utilité de la zone FCC. L'expérience a confirmé ces prédictions.

Le modèle [8] a non seulement prédit les frequences mais aussi très bien les intensités, voir figure 10. Pour le même vecteur $|q|=\left(\begin{array}{lll}0.25 & 0.25 & 0.25\end{array}\right)$ mais pour différentes positions de $\mathbf{Q}$ trois modes ont pu être observés, en accord avec les calculs de vecteurs propres. 


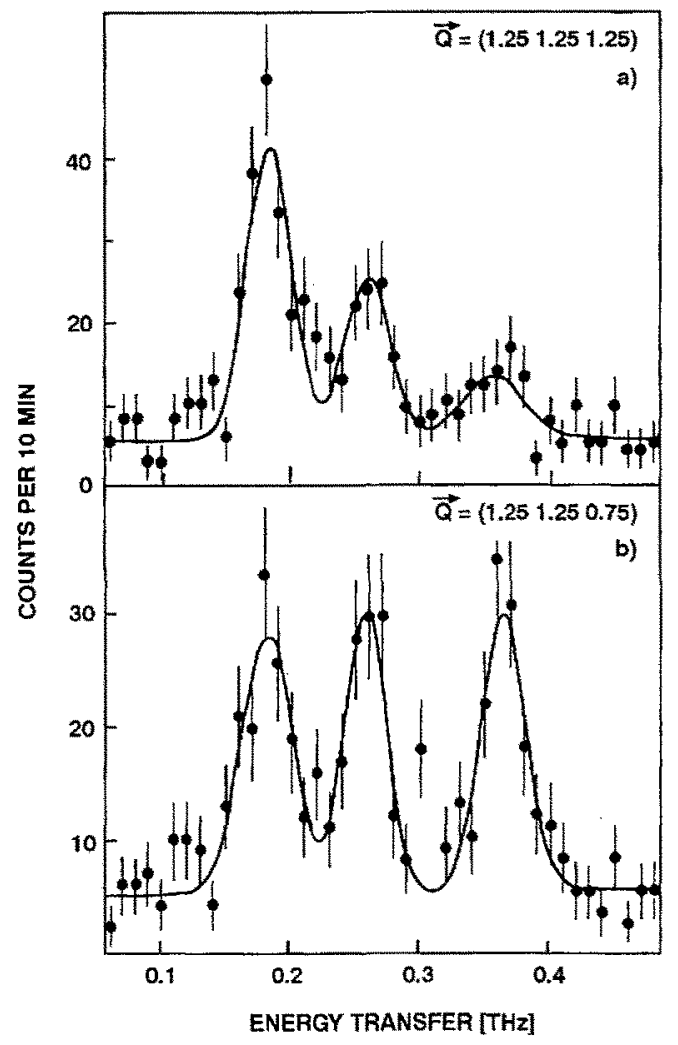

Figure 10: Scan à $Q$ constant pour $q=\left(\begin{array}{lll}0.25 & 0.25 & 0.25\end{array}\right)$ a) Trois différents modes petvent être observés. b) Un scan à $Q$ constant complémentaire. Les modes sont identiques qu'en a) par leur fréquence, longueur d'onde et vecteurs propres. Seule l'interférence avec les neutrons est différente, conduisant à une autre distribution des intensités en accord avec les calculs.

La structure magnétique des ions $\mathrm{Fe}^{3+}$ dans ce grenat est identique à celle de la configuration statique $(q=0)$, où les deux sous-systèmes ne sont pas couplés (voir figure 8). Un couplage apparait naturellement pour $\mathrm{q} \neq 0$ qui provoque une levée de la dégénérescence entre les deux modes initialement dégénérés de chaque sous-système.

Pour $\mathrm{q}=0$, une approche quantique [9] a permis de calculer le couplage induit par les fluctuations et a prédit l'existence d'un gap. Nous avons poussé la résolution du trois axes IN12 à l'ILL jusqu'à la limite, $\Delta \omega=0.06 \mathrm{meV}$ pour les magnons et $\Delta \omega=0.016 \mathrm{meV}$ pour le pic de Bragg. La largeur étroite pour ce dernier s'explique par le fait que la position de Bragg d'un bon cristal correspond à une fonction $\delta$ non seulement en énergie mais aussi en $\mathbf{Q}$. Nous avons alors mesuré des excitations magnétiques à $q=0$, correspondant à un gap de $\hbar \omega=0.14 \pm 0.02 \mathrm{meV}$ en présence d'un pic de Bragg antiferromagnétique très intense (voir figure 11). Il faut noter que les ajustements des courbes contiennent les corrections de résolution pour $\mathrm{k}_{1}=$ const et reproduisent très bien les intensités mesurées. 


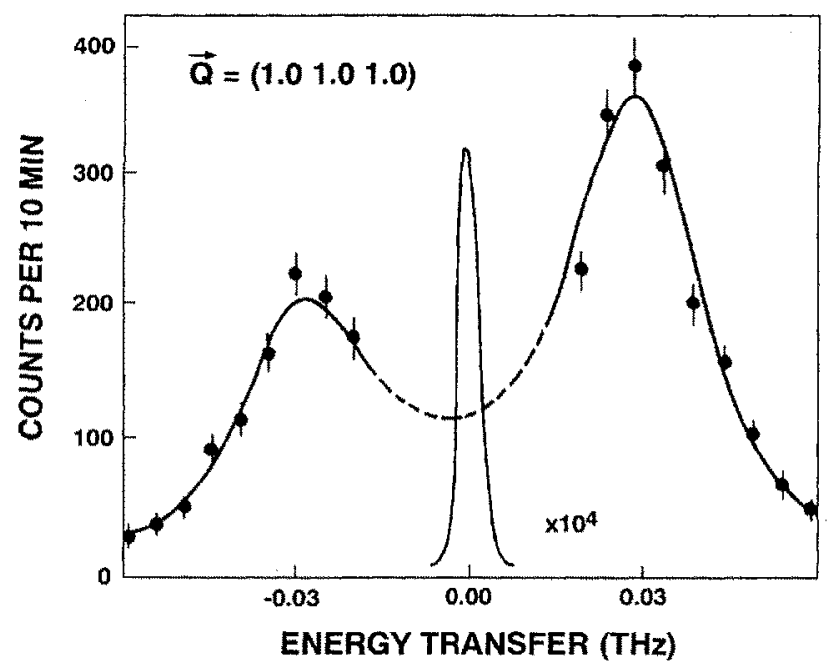

Figure 11: Scan à $Q$ constant à $q=0$. Le pic (111) antiferromagnétique est donné en échelle réduite.

\section{LES EXCITATIONS FERRO- ET ANTIFERROMAGNETIQUES}

Il est bien connu depuis longtemps que l'état fondamental d'un ferromagnétique avec tous les spins parallèles est un état quantique bien défini contrairement à l'état dit de Néel d'un antiferromagnétique simple. Néanmoins, l'état de Néel avec des spins up et down est une approximation qui a permis de calculer les courbes de dispersion d'ondes de spins et de nombreuses autres propriétés des antiferromagnétiques.

La figure 12 montre les courbes de dispersion d'une chaîne linéaire avec un couplage ferromagnétique ( $\mathrm{J}$ positif) et un couplage antiferromagnétique ( $\mathrm{J}$ négatif). La courbe de dispersion d'un ferromagnétique n'est pas dégénérée mais celle d'un antiferromagnétique l'est à cause de la symétrie par renversement du sens du temps. Les courbes de dispersion montrent que l'énergie maximum diffère d'un facteur 2 . 


\section{$H=-2 J \sum_{i} S_{i} S_{i+1}$ (Heisenberg System)}
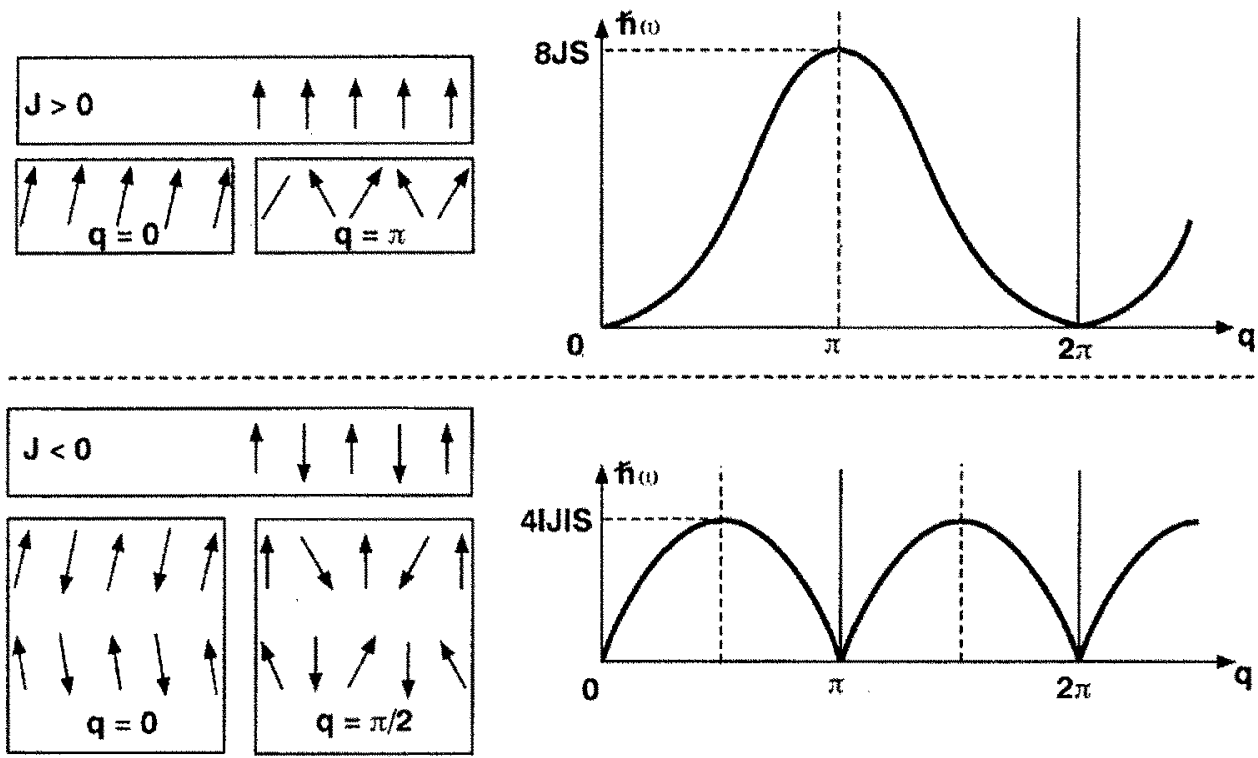

Figure 12: Les chaînes linéaires de spins (Heisenberg) avec des interactions d'échanges ferromagnétiques ( $J$ positif) ou antiferromagnétiques ( $J$ négatif) dans la limite classique. Pour $J>0$, l'arrangement parallèle correspond à l'état fondamental quantique. Pour $J<0, l$ l'état de Néel correspond à une visualisation utile mais pas à l'état fondamental. Il faut noter la différence d'énergie d'un facteur 2. Les courbes de dispersion antiferromagnétiques sont doublement dégénérées à cause de la symètrie par renversement du sens du temps. C'est-à-dire, une branche correspond à une precession vers la gauche et l'autre vers la droite. Les vecteurs propres sont donnés par leur projections sur un plan. Pour $q=\pi / 2$, dans le cas où $J<0$, un sous-réseau est au repos.

Les vecteurs propres associés aux énergies d'ondes de spin les plus grandes sont aussi données figure 12 en tant que projections des précessions dans un plan. Dans le cas d'un ferromagnétique, les spins voisins sont inclinés à l'opposé afin de créer l'énergie la plus grande possible. Tandis que dans le cas antiferromagnétique, les spins d'un même sous-réseau s'inclinent à l'opposé laissant l'autre sous-réseau au repos. L'autre mode dégénéré correspond à l'inverse. Il est clair que l'énergie correspondante est plus faible que celle du ferromagnétique. Un mode optique, où chaque sousréseau 's'incline à l'opposé de l'autre ne correspond pas à un mode propre d'un système antiferromagnétique simple.

\section{NOUVEAUX DÉVELOPPEMENTS SUR LES ANTIFERROMAGNÉTIQUES}

Dans les années 80 , de nouvelles idées pour des systèmes antiferromagnétiques $1 \mathrm{D}$ ont émergées. La conjecture de Haldane [10] pour $S=1$ (entier) a prẻdit un état fondamental singulet sous un état triplet dégénéré. Cela a été confirmé expérimentalement par M. Steiner et al d'un côté et L.P. Regnault et al d'un autre. Ceci ne sera pas discuté dans ce cours. 
Pour $\mathrm{S}=1 / 2$ (demi-entier), le spectre d'excitations a été prédit comme étant plus étendu à cause d'excitations appelées maintenant spinons [11]. Dans cette nouvelle description, le spectre des excitations apparaît comme un continuum compris entre une borne inférieure $\frac{\pi}{2} 4|J| S \sin (q a)$ et une bome supérieure $\frac{\pi}{2} 8|J| S \sin (q a / 2)$ (voir figure 13)

\section{ANTIFERROMAGNET with $S=1 / 2$}

\section{Néel-state

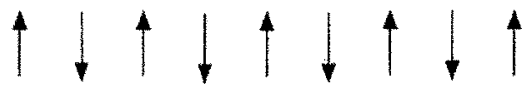

Spin flip $\Delta S= \pm 1$, due to neutron interaction
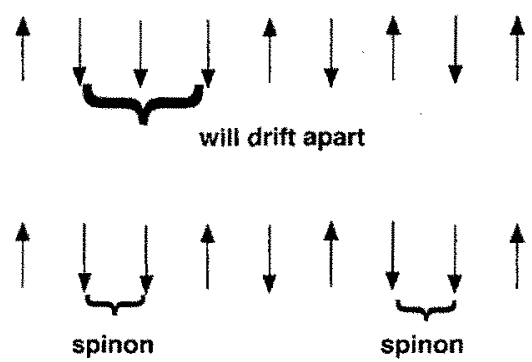

Dispersion spinon continuum

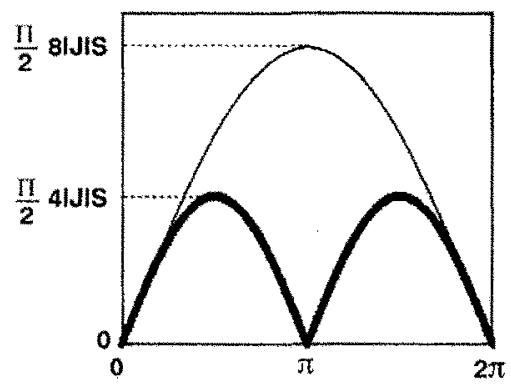

Figure 13: Les excitations appelées spinons apparaissent dans les chaînes antiferromagnétiques avec $S=1 / 2,3 / 2 \ldots$ Ces excitations ne sont pas des magnons, (ondes de spin planes) mais plutôt des combinaisons de magnons. Leur spectre est un continuum dans l'espace $\boldsymbol{Q}-\omega$. La limite basse de ce continuum est donné par la courbe de dispersion d'ondes de spin traditionnelle (courbe en gras) (voir aussi figure 12) et la limite haute par une fonction sin(qa/2) avec un maximum à $\pi$. Il faut noter que l'énergie la limite haute à $q=\pi$ est semblable à l'énergie des ondes de spin ferromagnétiques (voir figure 12). 


\section{SPECTRE DE SPINONS DANS $\mathrm{CuGeO}_{3}$}
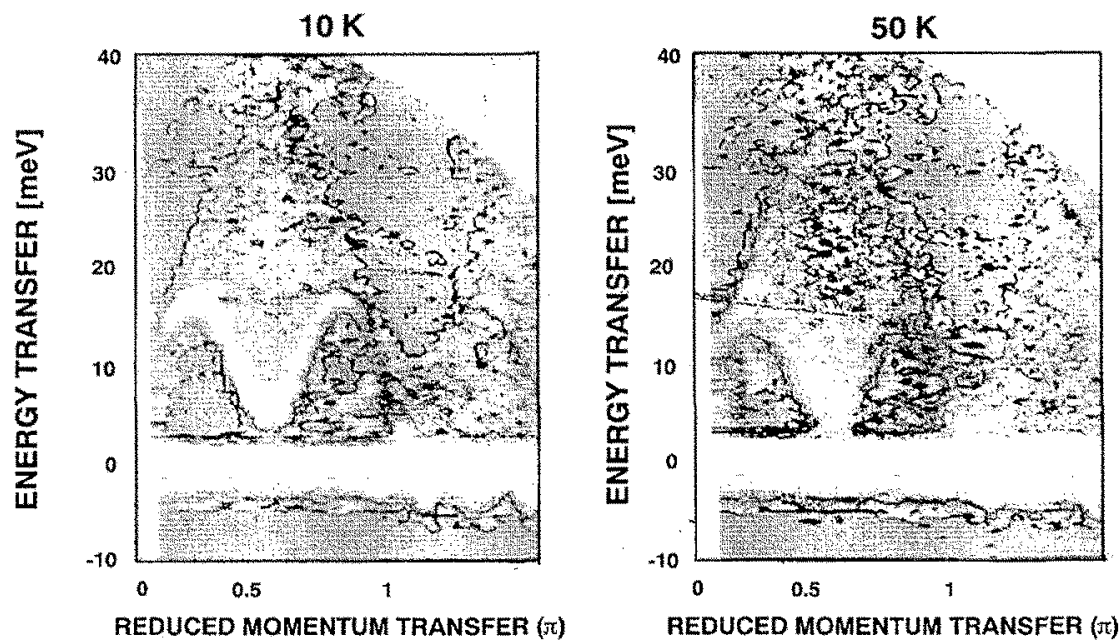

Figure 14: Le continuum des spinons dans $\mathrm{CuGeO}_{3}$ mesuré par temps de vol sur MARI à ISIS. Par comparaison avec la figure 13, la limite basse est donnée par la courbe de dispersion traditionnelle des ondes de spin antiferromagnétiques. A $10 \mathrm{~K}$, l'intensité de cette limite basse est augmentée à cause de la transition de phase spin-peierls à $14 K$.

Ces spinons ont été observés plusieurs fois. La première, à ma connaissance, a été réalisée dans $\mathrm{KCuF}_{3}[12]$ avec le spectromètre temps de vol (TOF) HET sur la source à spallation ISIS. II y a au moins deux raisons qui rendent le temps de vol intéressant sur les sources à spallation.

i) HET est un instrument construit pour les grands transferts d'énergie qui sont nécessaires pour $\mathrm{KCuF}_{3}$. Des énergies incidentes élevées sont en effet disponibles sur les sources à spallation.

ii) Le temps de vol a des avantages particuliers pour les systèmes 1-D où l'origine des courbes de dispersion dans l'espace réciproque par exemple le long de $z$, est un plan perpendiculaire à $z$ et non un point comme pour les systèmes 3-D. L'échantillon monocristallin peut être orienté par rapport au faisceau incident, $\mathrm{k}$, de telle sorte que chaque signal récolté dans les nombreux détecteurs peuvent être attribués à une composante $\mathrm{q}_{z}$ du vecteur $\mathbf{Q}$. Ici, $\mathrm{q}_{z}$ est la distance par rapport au plan d'origine le plus proche dans l'espace réciproque. Comme le transfert d'énergie possible est entièrement déterminé par $q_{z}$, de nombreux signaux peuvent etre projetés dans le plan $q_{z}-\omega$. De cette façon, on peut mesurer la courbe de dispersion d'un système 1-D le long de $q_{z}$. Un exemple particulièrement beau est l'étude du spectre de spinons dans $\mathrm{CuGeO}_{3}[13]$, voir Fig. 14. Dans ce système, le spectre a pu être déterminé sur plusieurs zones de Brillouin 


\section{SYSTÈME À NIVEAU FONDAMENTAL SINGULET}

Dans $\mathrm{CsFeCl}_{3}$ et $\mathrm{CsFeBr}_{3}$, l'ion $\mathrm{Fe}^{2+}$ de spin effectif $\mathrm{S}=1$ a un état fondamental singulet $(\mathrm{m}=0)$ à cause d'une anisotropie locale $\mathrm{D}\left(\mathrm{S}^{\mathrm{Z}}\right)^{2}$ équivalent à un splitting du champ cristallin. Le niveau excité local est un doublet $(m= \pm 1)$. Sans interactions d'échange, le spectre d'énergie serait une excitation à $\mathrm{D}$ sans dispersion en q (figure 15). La structure chimique de ces deux composés est identique à celle de $\mathrm{CsNiF}_{3}$ donnée en figure 3 . Ils ont une interaction d'échange $\mathrm{J}$ le long des chaînes et $\mathrm{J}^{\mathrm{l}}$ entre les chaines. Cormme $J \approx 10 \cdot J^{1}$, ce sont des systèmes quasiment $1-D$.
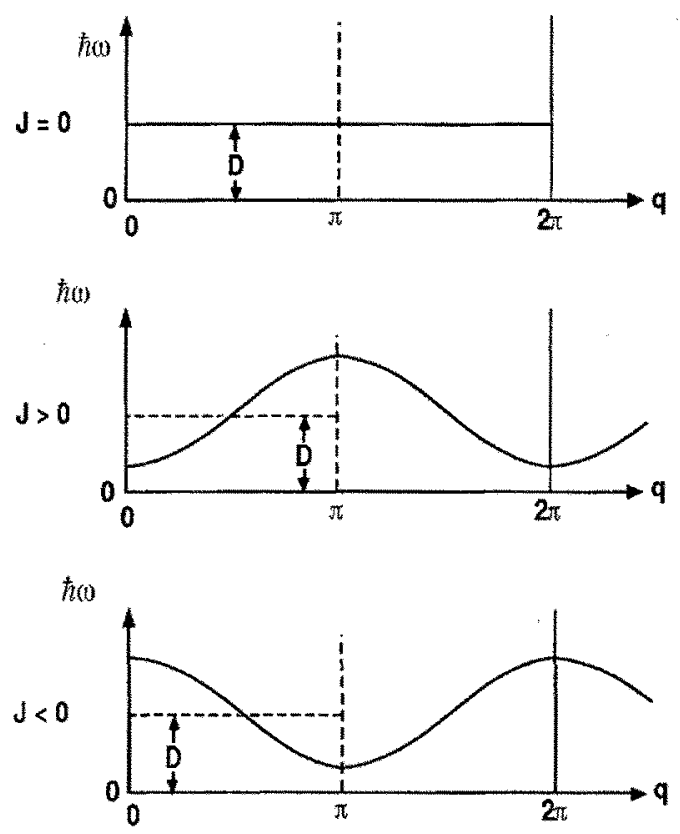

Figure 15 : dans certains systèmes 1-D de spin effectif $S=1$ comme $C s F e C l 3$ et $C s F e B r 3$, les ions $\mathrm{Fe}^{2+}$ subissent une anisotropie locale D pour des orientations par rapport à l'axe c qu'on pourrait appeler axe de quantification. Cette anisotropie induit une séparation entre le niveau fondamental singulet $m=0$ et l'état éxcité dégénéré $m= \pm 1$. L'intéraction d'échange $J$ induit une dispersion le long de qc qui module les fréquences autour de la valeur de D. Tant que D $>8|J|$, aucun ordre à longue distance apparaît et le système reste dans un état fondamental singulet.

Dans $\mathrm{CsFeCl}_{3}$, l'échange le long des chaînes est ferromagnétique avec un $\mathrm{J}$ positif. Par contre, dans $\mathrm{CsFeBr}_{3}$, l'échange est antiferromagnétique avec $\mathrm{J}<0$.

Le Hamiltonien s'écrit :

$$
\mathrm{H}=-2 J \sum_{i} \mathbf{S}_{i} \mathbf{S}_{i+1}-J^{1} \sum_{i, j} \mathbf{S}_{i} \mathbf{S}_{j}+D \sum_{i}\left(S_{i}^{2}\right)^{2}+g \mu_{B} m H_{e x}^{z} \sum S_{i}^{z}
$$

où $\mathrm{H}_{\text {ex }}^{z}$ est un champ appliqué le long de $z . g, \mu_{\mathrm{B}}$ et m sont respectivement le facteur de Landé, the magneton de Bohr et le nombre quantique $\mathrm{m}$ (ici \pm 1 ).

L'influence de $J$ est illustré figure 15 . Un $J$ positif induit des minima à $q=0,2 \pi, \ldots$ dans la courbe de dispersion, tandis qu'un I négatif, produit des minima à $q=\pi, 3 \pi, \ldots$ 
Les courbes de dispersion mesurées $[14,15]$ sont données figure 16. Même ̀̀ très basse température, les minima restent à fréquence finie et tout le système garde un état singulet fondamental $(\mathrm{m}=0)$ qui est macroscopiquement non magnétique. Pour cette raison, on a préféré appeler ces excitations magnétiques, excitons magnétiques et non ondes de spin.
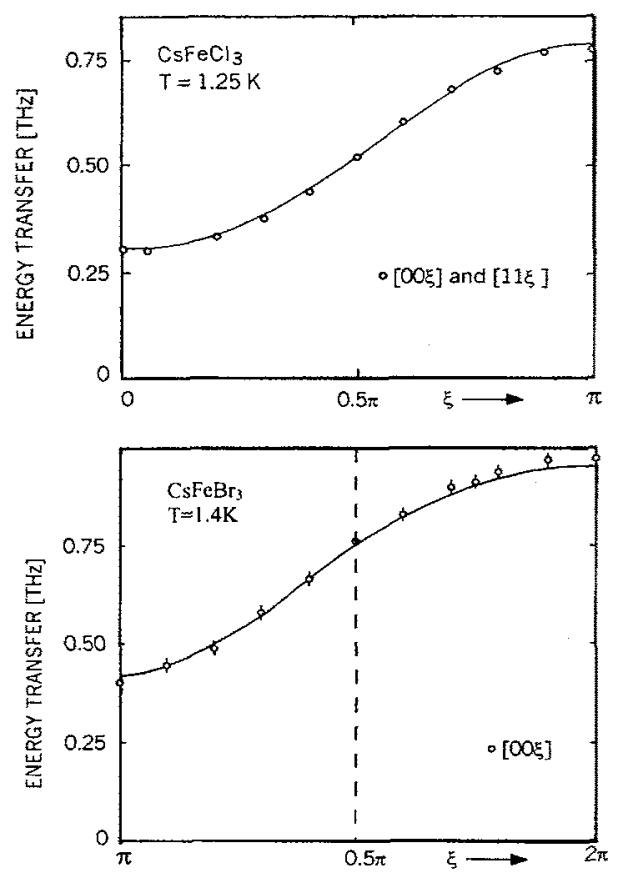

Fig. 16: courbes de dispersion des excitations magnétiques (excitons) dans $\mathrm{CsFeCl}$, avec $\mathrm{J}>0$, et dans $\mathrm{CsFeBr}$, avec $J<0$. Dans les deux cas, l'anisotropie locale D dépasse $8\lceil\mid$.

Dans les deux cas, $\mathrm{J}$ positif et négatif, les courbes de dispersion sont juste déplacées de $\pi$. Elles gardent la même amplitude et sont doublement dégénérées à cause de $m= \pm 1$. Cette double dégénérescence est différente de celle dans de simples antiferromagnétiques.

Certains détails ne sont pas discutés ici. Par exemple, $\mathrm{J}$ devient de plus en plus important avec la décroissance de la température car cela correspond à une augmentation du nombre $\mathrm{d}^{\text {'ions }} \mathrm{Fe}^{2+}$ dans l'état fondamental $\mathrm{m}=0$ [16].

Comme cela se voit sur le Hamiltonien ci-dessus, la dégénérescence due à $m= \pm 1$ peut être levée par un champ magnétique appliqué le long de z. Le splitting a été mesuré dans $\mathrm{CsFeBr}_{3}$ [14] et une transition de phase vers un ordre magnétique apparaît à 4.1 Tesla. (figure 17).

Cette expérience a demandé un champ magnétique horizontal pour observer le pic de surstructure magnétique en $(2 / 32 / 31)$. L'aimant disponible à ce moment là avait des bobines horizontales qui rendaient difficile le passage des neutrons lorsque l'échantillon était orienté de telle sorte que le champ magnétique fût parallèle à l'axe z. 


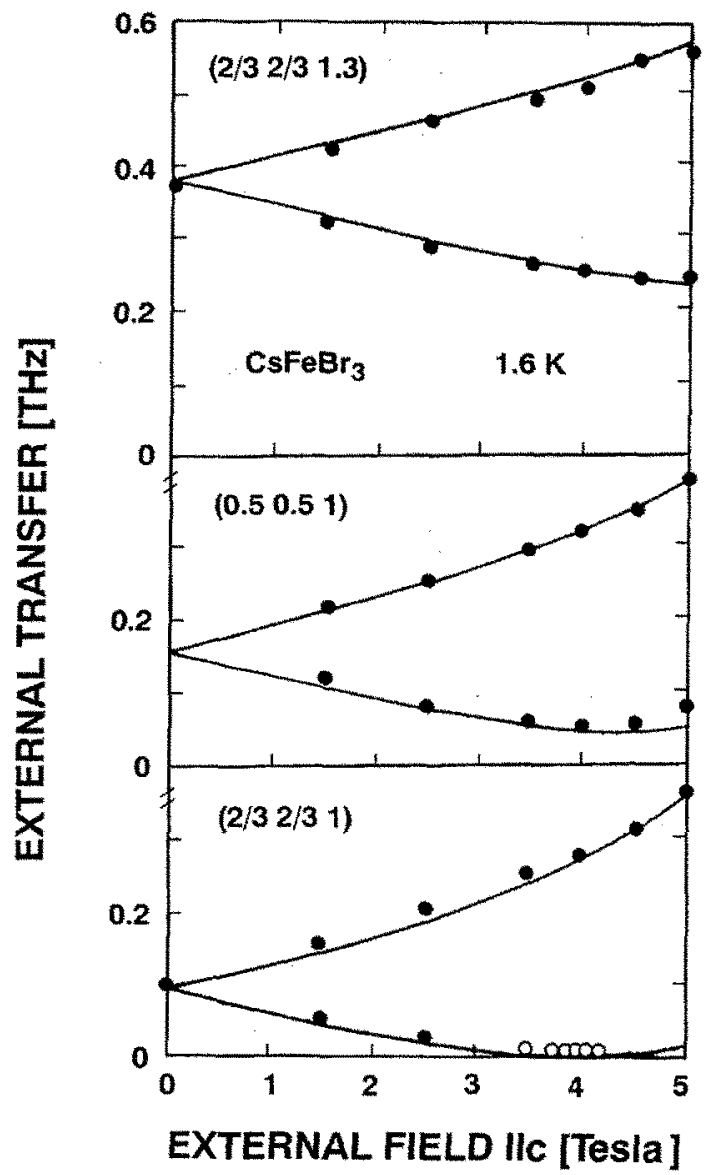

Figure 17: un champ extérieur appliqué le long de la direction z (axe de quantification) induit une séparation des excitations $m=+1$ et $m=-1$. Des pics de surstructure magnétiques apparaissent à 4.1 Tesla à $(2 / 32 / 3$ I) et révelent la transition de phase.

\section{CONCLUSIONS}

La diffusion de neutrons inélastique est un outil inégalé pour l'étude de la dynamique des systèmes magnétiques. Dans ce cours, seuls quelques exemples ont pu être données mais de très nombreux phénomènes sont étudiés avec des neutrons. Par exemple, nous n'avons pas abordé les observations des splittings de champ cristallin.

Les avantages des neutrons polarisés seront expliqués dans le cours de L.P. Regnault. 


\section{REFERENCES}

[1] B. Dorner, Acta Cryst. A28, 319 (1972).

[2] J. Kalus and B. Dorner, Acta Cryst. A29, 526 (1973).

[3] B. Dorner, "Coherent Inelastic Neutron Scattering in Lattice Dynamics" in Springer Tracts in Modern Physics, Ed. G. Höhler, Vol. 93, Springer Verlag Berlin, Heidelberg, New York (1982).

[4] M. Steiner and B. Dorner, Solid State Com. 12, 537 (1973).

[5] S.W. Lovesey, "Theory of Neutron Scattering from Condensed Matter", Clarendon Press, Oxford, England (1990).

[6] M. Steiner, B. Dorner and J. Villain, J. Phys. C: Solid State Phys. 8, 165 (1975).

[7] K. Kakurai, M. Steiner and B. Dorner, Europhys. Lett. 12, 653 (1990).

[8] Th. Brückel, B. Dorner, A.G. Gukasov, V.P. Plakhty, W. Prandl, E.F. Shender and O.P. Smimow, Z. Phys. B - Condensed Matter 72, 477 (1988).

[9] E. Shender, Zh. Eksp. Teor. Fiz. 83, 326 (1982) [Sov. Phys. JETP 56, 178 (1982)].

[10] F.D.M. Haldane, Phys. Rev. Lett. 50, 1153 (1983); Phys. Lett. 93 A, 464 (1983).

[11] G. Müller, H. Thomas, H. Beck and J.C. Bonner, Phys. Rev. B24, 1429 (1981);J.C. Bonner and H.W.J. Blöte, Phys. Rev. B25, 6959 (1982).

[12] S.E. Nagler, D.A. Tennant, R.A. Cowley, T.G. Perring and S.K. Satija, Phys. Rev. B44, 12361 (1991); D.A. Tennant, T.G. Perring, R.A. Cowley and S.E. Nagler, Phys. Rev. Lett. 70, 4003 (1993).

[13] M. Arai, M. Fujita, M. Motokawa, J. Akimitsu and S.M. Bennington, Phys. Rev. Lett. 77, 3649 (1996).

[14] B. Dorner, D. Visser and M. Steiner, Z. Phys. B - Condensed Matter 81, 75 (1990).

[15] B. Schmid, B. Dorner, D. Petitgrand, L.P. Regnault and M. Steiner, Z. Phys. B 95, 13 (1994).

[16] P.A. Lindgård, J. Phys. C 8, L178 (1975). 